\title{
Anomalous dynamics and equilibration in the classical Heisenberg chain
}

\author{
Adam J. McRoberts $\odot,{ }^{1}$ Thomas Bilitewski $\odot,{ }^{1,2,3}$ Masudul Haque, ${ }^{1,4,5}$ and Roderich Moessner ${ }^{1}$ \\ ${ }^{1}$ Max Planck Institute for the Physics of Complex Systems, Nöthnitzer Strasse 38, Dresden 01187, Germany \\ ${ }^{2}$ JILA, NIST, and Department of Physics, University of Colorado, Boulder, Colorado 80309, USA \\ ${ }^{3}$ Center for Theory of Quantum Matter, University of Colorado, Boulder, Colorado 80309, USA \\ ${ }^{4}$ Department of Theoretical Physics, Maynooth University, County Kildare, Ireland \\ ${ }^{5}$ Institut für Theoretische Physik, Technische Universität Dresden, Dresden 01062, Germany
}

(Received 27 August 2021; revised 14 February 2022; accepted 22 February 2022; published 4 March 2022)

\begin{abstract}
The search for departures from standard hydrodynamics in many-body systems has yielded a number of promising leads, especially in low dimensions. Here, we study one of the simplest classical interacting lattice models, the nearest-neighbor Heisenberg chain, with temperature as the tuning parameter. Our numerics expose strikingly different spin dynamics between the antiferromagnet, where it is largely diffusive, and the ferromagnet, where we observe strong evidence either of spin superdiffusion or an extremely slow crossover to diffusion. This difference also governs the equilibration after a quench, and, remarkably, is apparent even at very high temperatures.
\end{abstract}

DOI: 10.1103/PhysRevB.105.L100403

Introduction. Hydrodynamics has long been a cornerstone of our understanding of many-body systems, and has recently become the focus of renewed inquiry. Hydrodynamic phenomena of interest in low-dimensional quantum systems include equilibration [1,2], anomalous diffusion and transport [3-15], hydrodynamics and superdiffusion in long-range interacting systems [16,17], fracton and dipole-moment conserving hydrodynamics [18-20], generalized hydrodynamics in integrable quantum systems [21-33], and weak integrability breaking $[34,35]$. In addition, recent experimental studies are probing (emergent) hydrodynamics in interacting quantum spin models [16,36-38]. Hydrodynamics in classical manybody systems in low dimensions also poses many questions, perhaps most notably the appearance of anomalous diffusion and anomalous transport, often attributed to the Kardar-ParisiZhang (KPZ) universality class [39-54].

The focus of this Letter is the classical Heisenberg spin chain, for which the nature of hydrodynamics has provoked extensive debate. Based on the lack of integrability, it has been argued that ordinary diffusion holds for both spin and energy [55-61]. However, there have also been proposals of anomalous behavior [62-65], including an argument for logarithmically enhanced diffusion [65]. Reference [61], in contrast, has argued from a theory of non-Abelian hydrodynamics that each component of the spin follows a separate, ordinary diffusion equation.

Published by the American Physical Society under the terms of the Creative Commons Attribution 4.0 International license. Further distribution of this work must maintain attribution to the author(s) and the published article's title, journal citation, and DOI. Open access publication funded by the Max Planck Society.
In this Letter, we present a systematic numerical study of the dynamical correlations and equilibration dynamics over a wide range of temperatures, $T<|J|$ to $T=\infty$. We find ordinary diffusion of both spin and energy at $T=\infty$ and ordinary diffusion of energy at all (nonzero) temperatures in both the ferromagnetic (FM) and antiferromagnetic (AFM) chains [66]. Most strikingly, we find a qualitative difference between ferromagnetic and antiferromagnetic models at finite temperatures. This manifests as a temperature-dependent finite-time dynamical exponent in the spin correlations of the ferromagnetic chain, which departs from the diffusive exponent $\alpha=1 / 2$, whereas the antiferromagnetic chain displays behavior compatible with spin diffusion at all temperatures studied. This deviation is apparent even at high temperatures, where the correlation length is still of the order of a single lattice spacing - far from the low-temperature regime where the distinction between quadratic ferro- and linear antiferromagnetic spin-wave spectra may play a role. We have thus identified a, hitherto perhaps unappreciated, fundamental difference between the dynamics of the FM and AFM models.

The observed behavior of the ferromagnet could be interpreted as anomalous diffusion with a temperature-dependent exponent, or alternatively as a crossover at remarkably large timescales, rendering diffusion in practice unobservable experimentally for a wide range of temperatures. Intriguingly, at low temperatures where we obtain the best fit to a single power law, we observe the KPZ exponent almost perfectly across three decades in time. In addition, the spacetime profiles of correlation functions closely follow the KPZ scaling form. This establishes intermediate-time KPZ scaling at low temperatures in the FM Heisenberg model, even if ultimately followed by a crossover to normal diffusion at very long times.

As a related phenomenon, we study equilibration dynamics after quenches from an $X Y$ to a Heisenberg chain. Equilibration is shown to proceed via a power-law approach 
to the equilibrium value, with an exponent determined by that observed in the corresponding unequal-time equilibrium correlation function, again displaying anomalous finite-time exponents in the case of the FM.

Model. We consider the periodic-boundary classical Heisenberg chain, with Hamiltonian

$$
\mathcal{H}=-J \sum_{i=1}^{L} \boldsymbol{S}_{i} \cdot \boldsymbol{S}_{i+1}, \quad \boldsymbol{S}_{1}=\boldsymbol{S}_{L+1},
$$

for unit length classical spins $S_{i} \in S^{2}$. Here, $J=1$ for the FM chain, and $J=-1$ for the AFM chain. The dynamics is given by the classical Landau-Lifshitz equation of motion,

$$
\dot{\boldsymbol{S}}_{i}=\left\{\boldsymbol{S}_{i}, \mathcal{H}\right\}=J \boldsymbol{S}_{i} \times\left(\boldsymbol{S}_{i-1}+\boldsymbol{S}_{i+1}\right),
$$

which we solve numerically [66].

In equilibrium, we probe the spin-spin correlations

$$
\mathcal{C}^{S}(j, t)=\left\langle\boldsymbol{S}_{j}(t) \cdot \boldsymbol{S}_{0}(0)\right\rangle,
$$

and the energy correlations

$$
\mathcal{C}^{E}(j, t)=\left\langle E_{j}(t) E_{0}(0)\right\rangle-\mathcal{E}^{2},
$$

where $E_{j}=-J \boldsymbol{S}_{j} \cdot \boldsymbol{S}_{j+1}$ is the bond energy, and $\mathcal{E}=\langle E\rangle$ is the internal energy density. We use internal energy and temperature interchangeably, via $\mathcal{E}(T)=T-\operatorname{coth}(1 / T)[66,67]$. Also, the (equal-time) spin correlation length is

$$
\xi(\mathcal{E})=-1 / \log (-\mathcal{E}),
$$

which, as a function of $\mathcal{E}$, is the same for the Heisenberg (1) and $X Y$ chains (11) [66].

Both of these correlation functions are symmetric under parity and time reversal. To evaluate these correlations for a given $\mathcal{E}$, we first construct an ensemble of 20000 initial states drawn from the canonical ensemble of $\mathcal{H}$ at the temperature $T(\mathcal{E})[66,68]$. Each state is evolved in time [cf. (2)] with snapshots stored at intervals of $\Delta t=10 J^{-1}$. The correlation function at a fixed time difference $t$ is calculated by averaging over 1000 consecutive snapshots for every initial state.

Hydrodynamics and scaling functions. The hydrodynamic theory posits an asymptotic scaling form for the correlations of the conserved densities,

$$
\mathcal{C}(x, t) \sim t^{-\alpha} \mathcal{F}\left(t^{-\alpha} x\right),
$$

with a scaling exponent $\alpha$ and universal function $\mathcal{F}$.

The exponent is, in principle, independent of the precise form of $\mathcal{F}$, and may be extracted by fitting the autocorrelation function, $\mathcal{A}(t)=\mathcal{C}(0, t)$, to a power law,

$$
\mathcal{A}(t) \sim t^{-\alpha} .
$$

Ordinary diffusion corresponds to an exponent of $\alpha=1 / 2$, and a Gaussian scaling function,

$$
\mathcal{C}(x, t)=\frac{\chi}{(\pi D t)^{1 / 2}} \exp \left[-\left(\frac{x}{(D t)^{1 / 2}}\right)^{2}\right],
$$

where $\chi=\int d x \mathcal{C}(x, t)=\sum_{j} \mathcal{C}(j, t)$, and $D$ is the diffusion constant. This scaling function may be obtained directly by solving the ordinary diffusion equation.

The most well-known anomalous scaling is the KPZ universality class, with an exponent $\alpha=2 / 3$. There is no analytic form for the scaling function, but it is tabulated in Ref. [69].

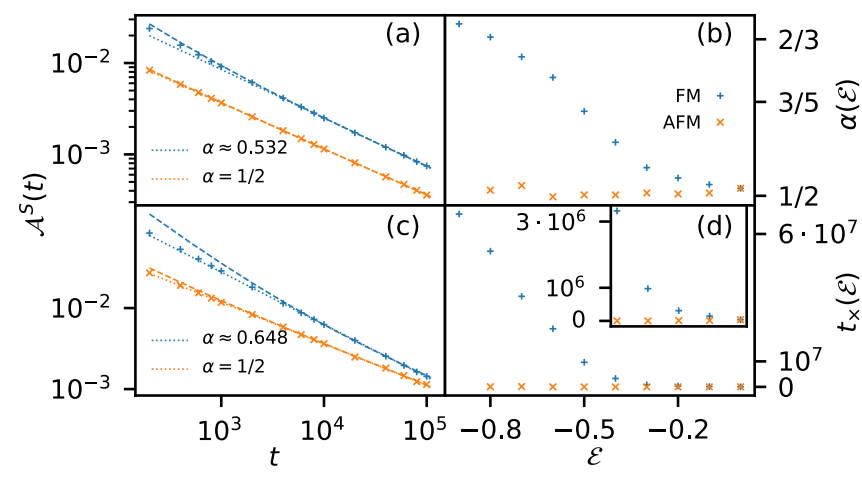

FIG. 1. Anomalous hydrodynamics in the FM: Power-law scaling of the autocorrelator $\mathcal{A}^{S}(t)$, and extracted exponents and crossover scales for the FM (blue, + ) and AFM (orange, $\times$ ). (a) and (c) show $\mathcal{A}^{S}(t)$ for $\mathcal{E}=-0.3$ and $\mathcal{E}=-0.7$, respectively. The dotted lines show the power-law fit (7), and the dashed lines show the finite-time corrected fit (9). (b) shows the estimated anomalous exponents, while (d) shows the diffusion crossover times estimated from (9)-the inset zooms in on the points $\mathcal{E}=-0.4$ to $\mathcal{E}=0$.

Even if the asymptotic behavior is diffusive, one might have finite-time corrections. The lowest-order correction to a diffusive autocorrelation function from non-Abelian hydrodynamics [61] is of the form

$$
\mathcal{A}(t) \sim(D t)^{-1 / 2}+\Lambda t^{-1} .
$$

From finite-time data, it may be difficult to distinguish this behavior from anomalous exponents [1].

Equilibrium correlations. We begin by examining the scaling exponent via the autocorrelation functions. We show $\mathcal{A}^{S}(t)$ in Figs. 1(a) and 1(c) for the FM and AFM at $\mathcal{E}=-0.3$ and $\mathcal{E}=-0.7$, for times $t=200$ to $t=10^{5}$.

The AFM displays ordinary spin diffusion, with the diffusive power law observable after a comparatively short time $t \approx 10^{3}$. The FM does not exhibit diffusion, at any finite temperature, over the timescales of our simulations.

The autocorrelations of the FM are, for these timescales, well approximated by a power law (7), with superdiffusive exponents [Fig. 1(b)]. One may also fit a crossover of the form (9). Adopting this point of view, we may extract a crossover time $t_{\times}(\mathcal{E})$ after which we would predict the system to show diffusion, via the effective exponent

$$
\alpha_{\text {eff }}(t)=-\frac{d \log [\mathcal{A}(t)]}{d \log (t)},
$$

with the crossover defined, arbitrarily, by $\alpha_{\text {eff }}\left(t_{\times}\right)=0.505$ (i.e., the time after which $\alpha_{\text {eff }}$ is sufficiently close to $1 / 2$ ). The estimated crossover times obtained for the FM are orders of magnitude larger than the AFM, reaching $t_{\times} \approx 10^{7} J^{-1}$ at low-to-intermediate temperatures [Fig. 1(d)].

In Fig. 2 we show the scaling collapses at these temperatures. The AFM is clearly consistent with a diffusive collapse (8); the FM is not. Since the autocorrelation function is well fit by an anomalous power law (7), we use these exponents to perform the scaling collapse in the FM. This collapses the correlations rather well, though there is some noise in the tails.

Moreover, despite the noise, one may observe that the tails of the correlations decay faster than a Gaussian. This suggests 


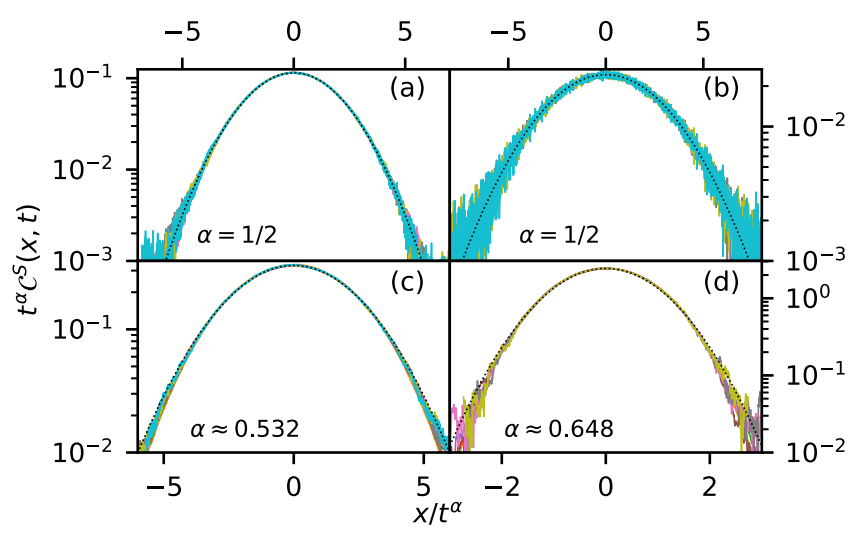

FIG. 2. Scaling collapses of the spin correlations $\mathcal{C}^{S}(x, t)$. Colors correspond to different fixed times from $t=2000$ up to $t=10^{5}$ $\left[t=8 \times 10^{4}\right.$ in (d)]. (a) and (b) show the diffusive collapse in the $\mathrm{AFM}$ at $\mathcal{E}=-0.3$ and $\mathcal{E}=-0.7$, respectively; (c) and (d) show anomalous collapses at $\mathcal{E}=-0.3$ and $\mathcal{E}=-0.7$ in the FM, with exponents $\alpha=0.532$ and $\alpha=0.648$, respectively. Dotted lines show a Gaussian scaling function as a guide to the eye.

that an enhancement of the diffusion constant alone [whether of the form (7), or a crossover (9)] is not the correct picture.

$K P Z$ scaling. We thus examine the possibility that we are observing a crossover from KPZ scaling. Indeed, the numerical evidence at low temperature is remarkably strong, shown in Fig. 3. The correlations up to $t=10^{4}$ collapse onto the KPZ function for $\mathcal{E}=-0.8$ and $\mathcal{E}=-0.9$. Beyond this time the noise in the tails is too great to reliably distinguish the form of the spatial decay, but the scaling exponent measured by the autocorrelation function is consistent with $\alpha=2 / 3$ up to the final time $t=10^{5}$. Moreover, at $\mathcal{E}=-0.8$, there are apparently no finite-time corrections to the power-law decay $\mathcal{A}(t) \sim t^{-2 / 3}$, for three decades in time.

Equilibration dynamics. In addition to our equilibrium simulations examining unequal-time correlations, we perform equilibration simulations probing the relaxation to thermal

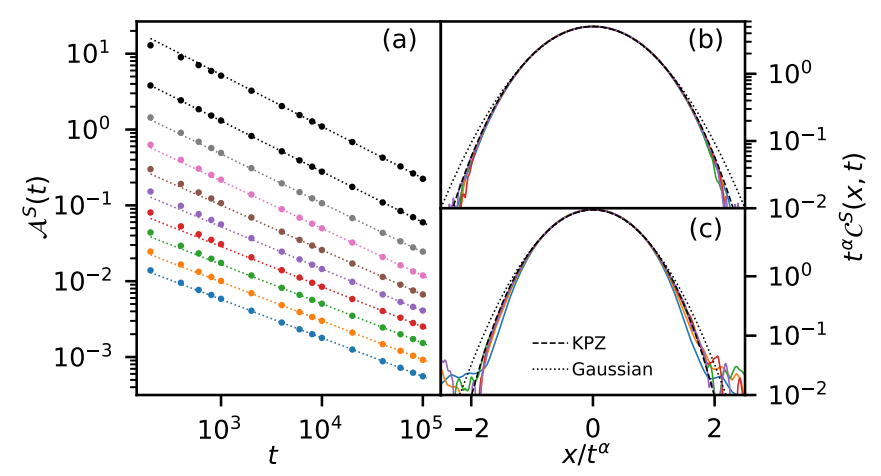

FIG. 3. Anomalous scaling in the FM. (a) shows the the autocorrelator $\mathcal{A}^{S}(t)$, vertically offset for clarity, for, in descending order, $\mathcal{E}=-0.9$ to $\mathcal{E}=0$ in steps of 0.1 . The power-law fits have the exponents of Fig. 1(b), except the black lines at $\mathcal{E}=-0.8$ and $\mathcal{E}=-0.9$, which are fit with the KPZ exponent $\alpha_{\mathrm{KPZ}}=2 / 3$. (b) and (c) show the scaling collapse using the extracted exponent comparing to the KPZ scaling function (dashed) and a Gaussian (dotted) at $\mathcal{E}=-0.8$ and $\mathcal{E}=-0.9$, respectively. equilibrium after a quench. This allows us to test whether the anomalous behavior, and the distinction between FM and AFM, are also observable in out-of-equilibrium dynamics.

We initially prepare the system in a thermal state of the $X Y$ chain,

$$
\mathcal{H}_{\mathrm{XY}}=-J \sum_{i=1}^{L} \boldsymbol{S}_{i} \cdot \boldsymbol{S}_{i+1}=-J \sum_{i=1}^{L} \cos \left(\phi_{i}-\phi_{i+1}\right),
$$

for unit length classical rotors $S_{i} \in S^{1}$. At time $t=0$, we quench the system, and evolve under the dynamics (2) of the Heisenberg chain. We examine the relaxation of the following observables:

$$
E^{\mu}(t)=-J\left\langle S_{i}^{\mu}(t) S_{i+1}^{\mu}(t)\right\rangle,
$$

which measures the energy attributed to the $\mu$ th spin components, and

$$
Q^{\mu}(t)=\left\langle S_{i}^{\mu}(t)^{2}\right\rangle
$$

which measures the total magnitude of the $\mu$ th spin components. These are natural measures of the anisotropy, which characterizes the relaxation from the initial state, satisfying $S_{i}^{z}=0 \forall i$, to a (quasi)thermal state of the isotropic Heisenberg chain. The equilibration of the energy fluctuations is measured using the heat capacity,

$$
C(t)=\frac{\left\langle\operatorname{var} E_{i}(t)\right\rangle}{T^{2}},
$$

where we take the spatial variance before the ensemble average to obtain a time-dependent quantity. As in the equilibrium simulations, we average over an ensemble of 20000 states, initially drawn from the canonical ensemble of $\mathcal{H}_{\mathrm{XY}}$.

We expect that the equilibration dynamics will be similarly hydrodynamic, since establishing the new global equilibrium requires the transport of conserved densities over long distances [1]. The relaxation of an observable $\mathcal{O}$ is therefore expected to follow a power law

$$
\delta \mathcal{O}(t):=\left|\mathcal{O}(t)-\mathcal{O}_{\text {eq }}\right|=\lambda t^{-\alpha},
$$

where $\mathcal{O}_{\text {eq }}$ is the thermal value of the observable in the Heisenberg chain.

These simulations exhibit complementary aspects of the same broad phenomenology observed in equilibrium. Figure 4 shows the equilibration dynamics at $\mathcal{E}=-0.5$. The extracted (anomalous) equilibration exponents have qualitatively similar dependence on energy as those extracted from equilibrium correlation functions [66]. The energy fluctuations, as measured by the heat capacity, always equilibrate diffusively. In the AFM, $E^{\mu}$ and $Q^{\mu}$ also show diffusive equilibration. In the FM, however, the equilibration of $E^{\mu}$ and $Q^{\mu}$ is anomalous. It should be noted that, although $E^{\mu}$ has dimensions of energy, it is, as $Q^{\mu}$, a measure of the magnetic anisotropy, and therefore equilibrates anomalously in the FM, rather than tracking the diffusive behavior of the energy fluctuations.

Thus, as in equilibrium, we observe a striking difference between the FM and AFM, with only the former displaying anomalous exponents.

While our simulations do not allow us to rule out a potential crossover to diffusive equilibration at even longer times, the observables can reasonably be described to have (fully) 


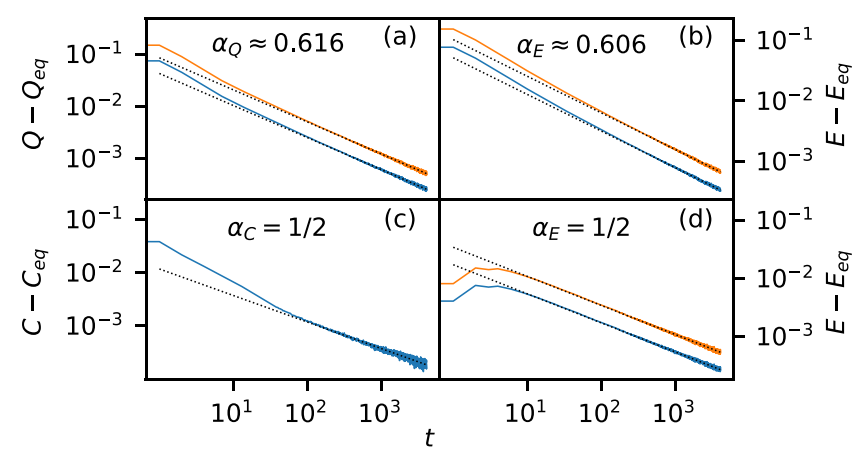

FIG. 4. Equilibration dynamics of $\mathcal{O}^{z}(t)-\mathcal{O}_{\text {eq }}^{z}$ (blue) and $\mathcal{O}^{\|}(t)-\mathcal{O}_{\text {eq }}^{\|}$(orange), where $\mathcal{O}^{\|}=\left(\mathcal{O}^{x}+\mathcal{O}^{y}\right) / 2$ is the average of the in-plane components. (a)-(c) show the equilibration of $Q, E$, and $C$ at $\mathcal{E}=-0.5$ in the $\mathrm{FM}$; (d) shows the equilibration of $E$ at $\mathcal{E}=-0.5$ in the AFM. $Q$ and $E$ appear to equilibrate with an anomalous exponent of $\alpha \approx 0.6$ in the FM, though the data are equally well described by a combination of power laws. The energy fluctuations (heat capacity) and the AFM equilibrate diffusively.

equilibrated with these anomalous exponents, in particular when considering a realistic experimental situation in which resolution and timescales might be limited.

Discussion and conclusions. We have conducted a detailed numerical study of the equilibrium and out-of-equilibrium dynamics of the classical Heisenberg chain, with large system sizes, simulation times, and range of temperatures. We find that, although ordinary diffusion is expected at infinite time, the FM exhibits a long-lived regime that is well described by an effective superdiffusive, temperature-dependent scaling exponent, with remarkably clean KPZ-like behavior at low temperature. The AFM, by contrast, swiftly evinces ordinary diffusion at all temperatures. The existence of such large intermediate scales is obviously relevant to experiments probing anomalous diffusion - anomalous behavior might be the only accessible experimental regime even when the longer-term behavior is diffusive.

A possible explanation of the intermediate-time regime and the stark difference between FM and AFM cases could be the presence of integrable ferromagnetic models, such as the continuum Landau-Lifshitz model [70-73] and the lattice FM model with $\log \left(1+\boldsymbol{S}_{i} \cdot \boldsymbol{S}_{i+1}\right)$ interactions [54,7377]. The Heisenberg FM studied in the present Letter could arguably be considered to be increasingly similar to either of these integrable models at lower temperatures. This would be consistent with our finding that the low-temperature FM behavior is closer to KPZ. Recent work [12-15] suggests the perturbative stability of KPZ scaling in systems which are close to integrability and preserve the rotational symmetry.

Nevertheless, it is remarkable that we observe an anomalous regime even at near-infinite temperatures, where the correlation length (5) is short, e.g., already less than a single lattice spacing at $\mathcal{E}=-0.3$. Intuitively, this regime does not seem to be close to either the continuum model or the integrable log-interaction model. This points to the need for a better understanding of how proximity to integrable points might play a role in the physics of the Heisenberg FM, especially at high temperatures.

A.J.McR. wishes to thank J. N. Hallen, and P. Suchsland for helpful discussions, and particularly B. A. Placke for explaining various aspects of $\mathrm{C}++$. This work was in part supported by the Deutsche Forschungsgemeinschaft (DFG) under Grant No. SFB 1143 (project-id 247310070) and the cluster of excellence ct.qmat (EXC 2147, project-id 390858490).
[1] J. Lux, J. Müller, A. Mitra, and A. Rosch, Hydrodynamic longtime tails after a quantum quench, Phys. Rev. A 89, 053608 (2014).

[2] E. Leviatan, F. Pollmann, J. H. Bardarson, D. A. Huse, and E. Altman, Quantum thermalization dynamics with matrixproduct states, arXiv:1702.08894.

[3] M. Ljubotina, M. Žnidarič, and T. Prosen, Spin diffusion from an inhomogeneous quench in an integrable system, Nat. Commun. 8, 16117 (2017).

[4] M. Ljubotina, M. Žnidarič, and T. Prosen, Kardar-Parisi-Zhang Physics in the Quantum Heisenberg Magnet, Phys. Rev. Lett. 122, 210602 (2019).

[5] F. Weiner, P. Schmitteckert, S. Bera, and F. Evers, Hightemperature spin dynamics in the Heisenberg chain: Magnon propagation and emerging Kardar-Parisi-Zhang scaling in the zero-magnetization limit, Phys. Rev. B 101, 045115 (2020).

[6] M. Fava, B. Ware, S. Gopalakrishnan, R. Vasseur, and S. A. Parameswaran, Spin crossovers and superdiffusion in the one-dimensional Hubbard model, Phys. Rev. B 102, 115121 (2020).

[7] M. Dupont and J. E. Moore, Universal spin dynamics in infinitetemperature one-dimensional quantum magnets, Phys. Rev. B 101, 121106(R) (2020).
[8] V. B. Bulchandani, C. Karrasch, and J. E. Moore, Superdiffusive transport of energy in one-dimensional metals, Proc. Natl. Acad. Sci. USA 117, 12713 (2020).

[9] D. Schubert, J. Richter, F. Jin, K. Michielsen, H. De Raedt, and R. Steinigeweg, Quantum versus classical dynamics in spin models: Chains, ladders, and square lattices, Phys. Rev. B 104, 054415 (2021).

[10] J. Richter and A. Pal, Anomalous hydrodynamics in a class of scarred frustration-free Hamiltonians, Phys. Rev. Research 4, L012003 (2022).

[11] M. Dupont, N. E. Sherman, and J. E. Moore, Spatiotemporal Crossover between Low- and High-Temperature Dynamical Regimes in the Quantum Heisenberg Magnet, Phys. Rev. Lett. 127, 107201 (2021).

[12] S. Gopalakrishnan and R. Vasseur, Kinetic Theory of Spin Diffusion and Superdiffusion in XXZ Spin Chains, Phys. Rev. Lett. 122, 127202 (2019).

[13] E. Ilievski, J. De Nardis, M. Medenjak, and T. Prosen, Superdiffusion in One-Dimensional Quantum Lattice Models, Phys. Rev. Lett. 121, 230602 (2018).

[14] E. Ilievski, J. De Nardis, S. Gopalakrishnan, R. Vasseur, and B. Ware, Superuniversality of superdiffusion, Phys. Rev. X 11, 031023 (2021). 
[15] J. De Nardis, S. Gopalakrishnan, R. Vasseur, and B. Ware, Stability of Superdiffusion in Nearly Integrable Spin Chains, Phys. Rev. Lett. 127, 057201 (2021).

[16] M. K. Joshi, F. Kranzl, A. Schuckert, I. Lovas, C. Maier, R. Blatt, M. Knap, and C. F. Roos, Observing emergent hydrodynamics in a long-range quantum magnet, arXiv:2107.00033.

[17] A. Schuckert, I. Lovas, and M. Knap, Nonlocal emergent hydrodynamics in a long-range quantum spin system, Phys. Rev. B 101, 020416(R) (2020).

[18] A. Gromov, A. Lucas, and R. M. Nandkishore, Fracton hydrodynamics, Phys. Rev. Research 2, 033124 (2020).

[19] J. Feldmeier, P. Sala, G. De Tomasi, F. Pollmann, and M. Knap, Anomalous Diffusion in Dipole- and Higher-MomentConserving Systems, Phys. Rev. Lett. 125, 245303 (2020).

[20] A. Morningstar, V. Khemani, and D. A. Huse, Kinetically constrained freezing transition in a dipole-conserving system, Phys. Rev. B 101, 214205 (2020).

[21] D. Bernard and B. Doyon, Conformal field theory out of equilibrium: A review, J. Stat. Mech.: Theory Exp. (2016) 064005.

[22] O. A. Castro-Alvaredo, B. Doyon, and T. Yoshimura, Emergent Hydrodynamics in Integrable Quantum Systems Out of Equilibrium, Phys. Rev. X 6, 041065 (2016).

[23] B. Bertini, M. Collura, J. De Nardis, and M. Fagotti, Transport in Out-of-Equilibrium XXZ Chains: Exact Profiles of Charges and Currents, Phys. Rev. Lett. 117, 207201 (2016).

[24] V. B. Bulchandani, R. Vasseur, C. Karrasch, and J. E. Moore, Solvable Hydrodynamics of Quantum Integrable Systems, Phys. Rev. Lett. 119, 220604 (2017).

[25] S. Sotiriadis, Equilibration in one-dimensional quantum hydrodynamic systems, J. Phys. A: Math. Theor. 50, 424004 (2017).

[26] E. Ilievski and J. De Nardis, Microscopic Origin of Ideal Conductivity in Integrable Quantum Models, Phys. Rev. Lett. 119, 020602 (2017).

[27] B. Doyon, H. Spohn, and T. Yoshimura, A geometric viewpoint on generalized hydrodynamics, Nucl. Phys. B 926, 570 (2018).

[28] B. Doyon, T. Yoshimura, and J.-S. Caux, Soliton Gases and Generalized Hydrodynamics, Phys. Rev. Lett. 120, 045301 (2018).

[29] V. B. Bulchandani, R. Vasseur, C. Karrasch, and J. E. Moore, Bethe-Boltzmann hydrodynamics and spin transport in the XXZ chain, Phys. Rev. B 97, 045407 (2018).

[30] B. Doyon, Lecture notes on generalised hydrodynamics, SciPost Phys. Lect. Notes 18 (2020).

[31] P. Ruggiero, P. Calabrese, B. Doyon, and J. Dubail, Quantum Generalized Hydrodynamics, Phys. Rev. Lett. 124, 140603 (2020).

[32] J. Dufty, K. Luo, and J. Wrighton, Generalized hydrodynamics revisited, Phys. Rev. Research 2, 023036 (2020).

[33] V. Alba, B. Bertini, M. Fagotti, L. Piroli, and P. Ruggiero, Generalized-hydrodynamic approach to inhomogeneous quenches: correlations, entanglement and quantum effects J. Stat. Mech.: Theory Exp. (2021) 114004.

[34] A. J. Friedman, S. Gopalakrishnan, and R. Vasseur, Diffusive hydrodynamics from integrability breaking, Phys. Rev. B 101, 180302(R) (2020).

[35] J. Lopez-Piqueres, B. Ware, S. Gopalakrishnan, and R. Vasseur, Hydrodynamics of nonintegrable systems from a relaxationtime approximation, Phys. Rev. B 103, L060302 (2021).

[36] C. Zu, F. Machado, B. Ye, S. Choi, B. Kobrin, T. Mittiga, S. Hsieh, P. Bhattacharyya, M. Markham, D. Twitchen, A.
Jarmola, D. Budker, C. R. Laumann, J. E. Moore, and N. Y. Yao, Emergent hydrodynamics in a strongly interacting dipolar spin ensemble, Nature (London) 597, 45 (2021).

[37] P. N. Jepsen, J. Amato-Grill, I. Dimitrova, W. W. Ho, E. Demler, and W. Ketterle, Spin transport in a tunable Heisenberg model realized with ultracold atoms, Nature (London) 588, 403 (2020).

[38] D. Wei, A. Rubio-Abadal, B. Ye, F. Machado, J. Kemp, K. Srakaew, S. Hollerith, J. Rui, S. Gopalakrishnan, N. Y. Yao, I. Bloch, and J. Zeiher, Quantum gas microscopy of KardarParisi-Zhang superdiffusion, arXiv:2107.00038.

[39] M. Kardar, G. Parisi, and Y.-C. Zhang, Dynamic Scaling of Growing Interfaces, Phys. Rev. Lett. 56, 889 (1986).

[40] H. Spohn, Exact solutions for KPZ-type growth processes, random matrices, and equilibrium shapes of crystals, Physica A 369, 71 (2006).

[41] T. Sasamoto and H. Spohn, Exact height distributions for the KPZ equation with narrow wedge initial condition, Nucl. Phys. B 834, 523 (2010).

[42] G. Amir, I. Corwin, and J. Quastel, Probability distribution of the free energy of the continuum directed random polymer in 1+1 dimensions, Commun. Pure Appl. Math. 64, 466 (2011).

[43] T. Kriecherbauer and J. Krug, A pedestrian's view on interacting particle systems, KPZ universality and random matrices, J. Phys. A: Math. Theor. 43, 403001 (2010).

[44] J. Quastel and H. Spohn, The one-dimensional KPZ equation and its universality class, J. Stat. Phys. 160, 965 (2015).

[45] H. van Beijeren, Exact Results for Anomalous Transport in One-Dimensional Hamiltonian Systems, Phys. Rev. Lett. 108, 180601 (2012).

[46] M. Kulkarni and A. Lamacraft, Finite-temperature dynamical structure factor of the one-dimensional Bose gas: From the Gross-Pitaevskii equation to the Kardar-Parisi-Zhang universality class of dynamical critical phenomena, Phys. Rev. A 88, 021603(R) (2013).

[47] S. G. Das, A. Dhar, K. Saito, C. B. Mendl, and H. Spohn, Numerical test of hydrodynamic fluctuation theory in the Fermi-Pasta-Ulam chain, Phys. Rev. E 90, 012124 (2014).

[48] H. Spohn, Nonlinear fluctuating hydrodynamics for anharmonic chains, J. Stat. Phys. 154, 1191 (2014).

[49] C. B. Mendl and H. Spohn, Equilibrium time-correlation functions for one-dimensional hard-point systems, Phys. Rev. E 90, 012147 (2014).

[50] M. Kulkarni, D. A. Huse, and H. Spohn, Fluctuating hydrodynamics for a discrete Gross-Pitaevskii equation: Mapping onto the Kardar-Parisi-Zhang universality class, Phys. Rev. A 92, 043612 (2015).

[51] C. B. Mendl and H. Spohn, Searching for the Tracy-Widom distribution in nonequilibrium processes, Phys. Rev. E 93, 060101(R) (2016).

[52] Z. Chen, J. de Gier, I. Hiki, and T. Sasamoto, Exact Confirmation of 1D Nonlinear Fluctuating Hydrodynamics for a Two-Species Exclusion Process, Phys. Rev. Lett. 120, 240601 (2018).

[53] S. Lepri, R. Livi, and A. Politi, Too close to Integrable: Crossover From Normal to Anomalous Heat Diffusion, Phys. Rev. Lett. 125, 040604 (2020).

[54] A. Das, M. Kulkarni, H. Spohn, and A. Dhar, Kardar-ParisiZhang scaling for an integrable lattice Landau-Lifshitz spin chain, Phys. Rev. E 100, 042116 (2019). 
[55] R. W. Gerling and D. P. Landau, Comment on "Anomalous Spin Diffusion in Classical Heisenberg Magnets", Phys. Rev. Lett. 63, 812 (1989).

[56] R. W. Gerling and D. P. Landau, Time-dependent behavior of classical spin chains at infinite temperature, Phys. Rev. B 42, 8214 (1990).

[57] M. Böhm, R. W. Gerling, and H. Leschke, Comment on "Breakdown of Hydrodynamics in the classical 1D Heisenberg model", Phys. Rev. Lett. 70, 248 (1993).

[58] N. Srivastava, J.-M. Liu, V. Viswanath, and G. Müller, Spin diffusion in classical Heisenberg magnets with uniform, alternating, and random exchange, J. Appl. Phys. 75, 6751 (1994).

[59] V. Oganesyan, A. Pal, and D. A. Huse, Energy transport in disordered classical spin chains, Phys. Rev. B 80, 115104 (2009).

[60] D. Bagchi, Spin diffusion in the one-dimensional classical Heisenberg model, Phys. Rev. B 87, 075133 (2013).

[61] P. Glorioso, L. V. Delacrétaz, X. Chen, R. M. Nandkishore, and A. Lucas, Hydrodynamics in lattice models with continuous non-Abelian symmetries, SciPost Phys. 10, 15 (2021).

[62] G. Müller, Anomalous Spin Diffusion in Classical Heisenberg magnets, Phys. Rev. Lett. 60, 2785 (1988).

[63] O. F. de Alcantara Bonfim and G. Reiter, Breakdown of Hydrodynamics in the Classical 1D Heisenberg Model, Phys. Rev. Lett. 69, 367 (1992).

[64] O. F. de Alcantara Bonfim and G. Reiter, De Alcantara Bonfim and Reiter Reply, Phys. Rev. Lett. 70, 249 (1993).

[65] J. De Nardis, M. Medenjak, C. Karrasch, and E. Ilievski, Universality Classes of Spin Transport in One-dimensional Isotropic Magnets: The Onset of Logarithmic Anomalies, Phys. Rev. Lett. 124, 210605 (2020).

[66] See Supplemental Material at http://link.aps.org/supplemental/ 10.1103/PhysRevB.105.L100403 for additional details on the exact thermodynamics, the numerical methods, including the construction of thermal states and time evolution, results on the infinite temperature case, the energy correlations, and the equilibration dynamics and extracted equilibration exponents.

[67] M. E. Fisher, Magnetism in one-dimensional systems-the Heisenberg model for infinite spin, Am. J. Phys. 32, 343 (1964).

[68] D. Loison, C. Qin, K. Schotte, and X. Jin, Canonical local algorithms for spin systems: Heat bath and Hasting's methods, Eur. Phys. J. B 41, 395 (2004).

[69] M. Prähofer and H. Spohn, Exact scaling functions for onedimensional stationary KPZ growth, J. Stat. Phys. 115, 255 (2004).

[70] M. Lakshmanan, Continuum spin system as an exactly solvable dynamical system, Phys. Lett. A 61, 53 (1977).

[71] L. Takhtajan, Integration of the continuous Heisenberg spin chain through the inverse scattering method, Phys. Lett. A 64, 235 (1977).

[72] H. C. Fogedby, Solitons and magnons in the classical Heisenberg chain, J. Phys. A: Math. Gen. 13, 1467 (1980).

[73] L. Faddeev and L. Takhtajan, Hamiltonian Methods in the Theory of Solitons (Springer, Berlin, 1987).

[74] Y. Ishimori, An integrable classical spin chain, J. Phys. Soc. Jpn. 51, 3417 (1982).

[75] E. K. Sklyanin, Classical limits of the SU(2)-invariant solutions of the Yang-Baxter equation, J. Sov. Math. 40, 93 (1988).

[76] E. K. Sklyanin, Some algebraic structures connected with the Yang-Baxter equation, Funct. Anal. Appl. 16, 263 (1982).

[77] T. Prosen and B. Žunkovič, Macroscopic Diffusive Transport in a Microscopically Integrable Hamiltonian System, Phys. Rev. Lett. 111, 040602 (2013). 